\title{
Localization of Sparse Transmural Excitation Stimuli from Surface Mapping
}

\author{
Jingjia Xu, Azar Rahimi Dehaghani, Fei Gao, and Linwei Wang \\ Computational Biomedicine Laboratory, Golisano College of Computing and \\ Information Sciences, Rochester Institute of Technology, Rochester, NY, 14623, USA
}

\begin{abstract}
As in-silico 3D electrophysiological (EP) models start to play an essential role in revealing transmural EP characteristics and diseased substrates in individual hearts, there arises a critical challenge to properly initialize these models, i.e., determine the location of excitation stimuli without a trial-and-error process. In this paper, we present a novel method to localize transmural stimuli based on their spatial sparsity using surface mapping data. In order to overcome the mathematical ill-posedness caused by the limited measurement data, a neighborhoodsmoothness constraint is used to first obtain a low-resolution estimation of sparse solution. This is then used to initialize an iterative, re-weighted minimum-norm regularization to enforce a sparse solution and thereby overcome the physical ill-posedness of the electromagnetic inverse problem. Phantom experiments are performed on a human heart-torso model to evaluate this method in localizing excitation stimuli at different regions and depths within the ventricles, as well as to test its feasibility in differentiating multiple remotely or close distributed stimuli. Real-data experiments are performed on a healthy and an infarcted porcine heart, where activation isochronous simulated with the reconstructed stimuli are significantly closer to the catheterized mapping data than other stimuli configurations. This method has the potential to benefit the current research in subject-specific EP modeling as well as to facilitate clinical decisions involving device pacing and ectopic foci.
\end{abstract}

Keywords: transmural electrophysiology, in silico electrophysiological models, sparse excitation stimuli, surface mapping.

\section{Introduction}

Disruption to the path of electrical propagation in the heart can lead to lifethreatening situations and sudden cardiac death 11. However, current clinical routines measure cardiac electrophysiological (EP) activity by surface mapping data, i.e., body-surface electrocardiograms (ECG) or heart-surface catheterized mapping, which provides a poor surface surrogate for transmural EP activities across the depth of the myocardium [2]. Recent research has seen promising progress towards this challenge, where these surface mapping data are combined with 3D in silico EP models via different mathematical methods to get an estimation of the individualized transmural EP characteristics, such as the maximum 
a posteriori (MAP) of 3D action potential 34 and the estimation of conduction velocity [514. These different methods of subject-special EP modeling have the potential to improve the early diagnosis and prevention of malignant arrhythmia, and to predict patient response to different therapeutic interventions.

However, these studies face the common challenge of properly initializing the EP models, i.e., setting the locations of excitation stimuli, which will directly impact the calibration of EP models. In diseased ventricles, such as hearts with focal arrhythmia, the excitation may not start at regular Purkinje end-terminals. Even in healthy ventricles, there is a lack of practical means to measure the locations of subject-specific Purkinje end-terminals that may vary from subject to subject. How to obtain accurate locations for the ventricular stimuli becomes a critical issue for subject-specific EP modeling, and some initial efforts include personalizing Purkinje end-terminals from surface optical mapping data 6 .

Excitation stimuli, the starting points of electrical propagation, are typically sparse in space no matter whether the ventricles are under sinus-rhythm or pacing (external stimulus, foci, etc) conditions. In this paper, we exploit this spatial sparsity of EP stimuli to solve the problem of transmural stimuli localization using surface mapping data. We present a two-step algorithm where a neighborhood-smoothness constrained minimum-norm solution is first obtained to overcome the mathematical ill-posedness of the problem caused by the limited number of surface data. This solution provides a low-resolution estimation of the spatial distribution of excitation stimuli, which is then used to initialize an iterative re-weighted regularization method that, based on the stimuli sparsity in space, prunes the initial estimation of the stimuli into a sparse solution that pinpoints the locations of stimuli. Phantom experiments are perform on a human heart-torso model to evaluate this method in localizing single stimulus $(n=175)$ at different longitudinal, circumferential, and transmural regions within the ventricles and to further test its feasibility in differentiating multiple, closely or remotely distributed stimuli. Real-data experiments are performed on a healthy and an infarcted porcine heart, where the activation isochrones simulated with the reconstructed stimuli are significantly more consistent with the measured catheter maps than other stimuli configurations. Pinpointing transmural excitation stimuli from noninvasive or minimally-invasive surface data, this method is potentially beneficial to subject-specific EP modeling research as well as clinical decisions involving external device pacing and ectopic foci.

\section{Method}

The relationship between the excitation stimuli and surface mapping data can be described by a quasi-static simplification of Maxwell's equation on an imagederived discrete, anatomically-detailed model for any individual subject [7]:

$$
\Phi=\mathbf{H u}+\mathbf{n}
$$

where $\boldsymbol{\Phi}_{m \times 1}$ represents the surface mapping potential and $\mathbf{u}_{n \times 1}$ the value of active electrical sources distributed within the 3D myocardium. At the begin 
of electrical propagation, only very few locations are excited while the majority of the myocardium are at the resting stage, i.e., $\mathbf{u}$ is a sparse vector where the majority of the elements has the value 0 . Transfer matrix $\mathbf{H}_{m \times n}(m \ll n)$ encodes the geometrical and conductivity information of the individual. $\mathbf{n}$ is added to represent uncertainty caused by measurement and modeling error.

The inverse problem of inferring $\mathbf{u}$ from $\boldsymbol{\Phi}$ is plagued by two types of nonuniqueness [7, and we present a two-step algorithm to overcome the two challenges step by step:

1. First, to overcome the mathematical ill-posedness caused by the limited number of surface data compared to the number of unknowns in the heart, a neighborhood-smoothness constraint is imposed to achieve the effect of reducing the number of unknowns and to obtain a low-resolution solution of $\hat{\mathbf{u}}$. At this moment, the solution $\hat{\mathbf{u}}$ is not sparse.

2. Second, this inverse problem is further afflicted with the physical ill-posedness associated with the electromagnetic field, where adjacent stimuli can produce the same potential distribution on the surface. To overcome this problem, we present a sparsity-based, iterative re-weight minimum-norm regularization which, initialized by the solution from the first step, will pinpoint the exact location of excitation stimuli by achieving a sparse solution of $\hat{\mathbf{u}}$.

Neighborhood-Smoothness Estimation: The inverse solution derived at the first step overcomes the mathematical ill-posedness by imposing a neighborhoodsmoothness constraint [8] on a minimum norm cost function. It thus considers the neighborhood as one region to reduce the number of unknown variables:

$$
\min _{\mathbf{u}}\|\mathcal{S} \mathbf{u}\|^{2}, \quad \text { s.t }:\|\mathbf{\Phi}-\mathbf{H u}\| \leq \sigma^{2}
$$

where $\mathcal{S}$ is the neighborhood-smoothness term and $\sigma^{2}$ the variance of the noise.

The design of $\mathcal{S}=\boldsymbol{\Omega} \otimes \mathbf{I} \Psi$ takes into account two factors: The first factor (diagonal weight matrix $\boldsymbol{\Omega}$ ) considers the contribution of each region to the surface recordings: $\Omega_{i i}=\sqrt{\mathbf{h}_{i,:} \mathbf{h}_{i,:}^{T}}, \mathbf{h}_{i,:}$ being the $i$-th row of the forward mapping matrix $\mathbf{H}$; The second factor $\boldsymbol{\Psi}=\left(\psi_{1}^{T}, \psi_{2}^{T}, \ldots, \psi_{n}^{T}\right)^{T}$ decides the size of neighborhood for the smoothness constraint and is defined as:

$$
\psi_{i}=\frac{1}{N^{2}}\left(\frac{\sum_{b} \varphi_{b}}{\sum_{b} \varphi_{b}+1}-\varphi_{i}\right), \quad \text { s.t }:\left\|\mathbf{r}_{i}-\mathbf{r}_{b}\right\| \leq N
$$

where $\varphi$ is value of $\mathbf{u}$ weighted by $\boldsymbol{\Omega}$, and $\mathbf{r}_{i}$ and $\mathbf{r}_{b}$ are the coordinates for the nodes. In other words, we define the spatial neighborhood by radius $N$, combined with boundary conditions so that boundary points only have a partial sphere.

Sparse Source Reconstruction: Within the "smoothest" solution from first step as a priori, the sparse stimuli is reconstructed in the second step with a re-weight minimum norm algorithm [9] that performs an iterative procedure of energy localization to overcome the physical ill-posedness problem:

$$
\min _{\mathbf{q}_{k}}\left\|\mathbf{q}_{k}\right\|, \quad \text { s.t : }\left\|\mathbf{H} \mathbf{W}_{k} \mathbf{q}_{k}-\mathbf{\Phi}\right\| \leq \sigma^{2}
$$




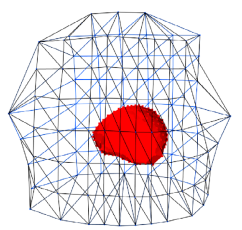

(b)

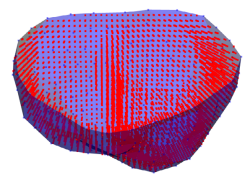

(a)

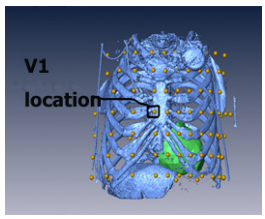

(c)

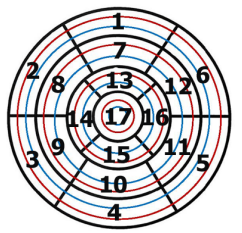

(d)

Fig. 1. (a) and (b): Boundary-element represented torso model with coupled meshfree nodes represented ventricular model; (c) Body-surface 120 leads with electrodes covering both the front and back of the body surface; (d) $17 \times 3$ segment of the LV where 17 is the standard AHA segments plus 3 transmural layers

where a weighted minimum-norm $\left\|\mathbf{q}_{k}=\mathbf{W}_{k}^{-1} \mathbf{u}_{k}\right\|$ is performed in each iteration, and the diagonal weight matrix $\mathbf{W}_{k}$ at iteration $k$ has elements from the previous solution $\mathbf{u}_{k-1}$. The relatively large entries in $\mathrm{W}$ reduce the contribution of the corresponding elements of $\mathbf{u}$ in the cost function, and vice versa. By an iterative procedure, a sparse solution is achieved by adjust the weight matrix and, as a result, the most-likely candidate of stimuli will have its signal value strengthened when the rest candidates will have their values weakened to 0 .

This optimization problem (4) is solved through an adaptive regularization:

$$
\mathbf{u}_{k}=\operatorname{diag}\left(\mathbf{u}_{k-1}\right) \cdot \arg \min _{\mathbf{q}_{k}}\left\{\left\|\mathbf{\Phi}-\mathbf{H} \operatorname{diag}\left(\mathbf{u}_{k-1}\right) \mathbf{q}_{k}\right\|_{2}+\lambda\left\|\mathbf{q}_{k}\right\|\right\}
$$

where $\lambda$ is a regularization parameter, determined by L-curve in this study.

Combining the two-step process, the initial $\mathbf{u}_{0}$ is provided by neighborhoodsmoothness solution to start the iterative, re-weighted minimum norm regularization. The iteration terminates when the difference between two successive solutions is smaller than the pre-defined tolerance, and a sparse and unique distribution of excitation stimuli will be achieved.

\section{Results}

\subsection{Phantom Experiment}

Phantom experiments are conducted on a human anatomical heart-torso model, derived from $3 \mathrm{~mm}$ whole-body CT scans. Fig. 1 shows the heart-torso model(a) with coupled ventricular model(b); (c) shows the set up of body-surface 120 leads which cover both the front and back of the body surface. We evaluate our method on 1) localizing single stimulus at different locations and depths of the ventricles, and 2) localizing and differentiating multiple stimuli distributed close or remotely with each other. In each case, true stimuli locations are set to simulate 120-lead body-surface ECG, which are then corrupted with 20-dB white Gaussian noise as inputs for localizing the stimuli. Localization errors are quantified by the Euclidean distance between the reconstructed and true stimuli. 


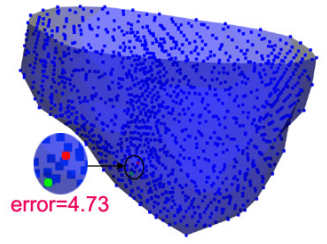

(a) Epicardial Stimulus

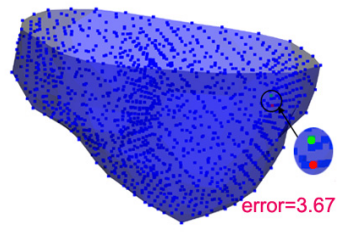

(b) Lateral Stimulus

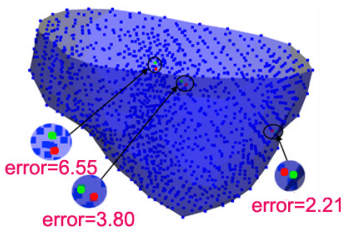

(c)Multi-stimuli

Fig. 2. (a) Single stimulus in epicardial-septal LV. (b) Single stimulus in endocardiallateral LV. (c) 3 remotely distributed stimuli at septal-endocardial and lateralintramural LV: average error $=3.58 \mathrm{~mm}$.

Table 1. Accuracy of Single Stimulus Localization at Different Regions

\begin{tabular}{|c|c|c|c|c|c|c|}
\hline Segment & $\begin{array}{c}\text { Anterior } \\
(\mathrm{n}=28)\end{array}$ & $\begin{array}{c}\text { Inferior } \\
(\mathrm{n}=28)\end{array}$ & $\begin{array}{c}\text { Apex } \\
(\mathrm{n}=6)\end{array}$ & $\begin{array}{c}\text { Lateral } \\
(\mathrm{n}=49)\end{array}$ & $\begin{array}{c}\text { Septal } \\
(\mathrm{n}=49)\end{array}$ & $\begin{array}{c}\text { RV } \\
(\mathrm{n}=15)\end{array}$ \\
\hline Error $(\mathrm{mm})$ & $0.11 \pm 0.60$ & $0.23 \pm 0.85$ & $0.53 \pm 1.30$ & $0.56 \pm 1.72$ & $1.05 \pm 2.46$ & $2.44 \pm 4.34$ \\
\hline Missed \% & $3.57 \%$ & $0 \%$ & $0 \%$ & $2.04 \%$ & $0 \%$ & $6.67 \%$ \\
\hline
\end{tabular}

A. Single Stimulus of Different Locations: To evaluate the accuracy in localizing stimulus at different locations of the ventricles, we consider the location by $17 \times 3$ segment of the LV where 17 is the standard AHA segments along longitudinal and circumferential directions plus 3 transmural layers (epicardial, endocardial, and intramural) (Fig.1(d)). By selecting a single excitation stimulus in these 51 segments, with 3-4 cases in each segment, altogether we have 160 cases of single stimulus covering different areas of LV and 15 randomly selected cases within the RV. For all the 175 cases, the neighborhood radius $\mathrm{N}$ is set to be $7 \mathrm{~mm}$ and the iterative regularization takes in average 6.2 iterations to converge. Fig. 2 shows two examples of a single stimulus at the epicardial-septal LV (a) and at the endocardial-lateral LV (b), with superimposed true stimuli (red) and reconstructed stimuli (green). Table 1 compares the accuracy of stimuli location at different ventricular regions, and Table 2 compares the accuracy of stimuli location at different transmural layers. Note missed case means that the reconstructed $\hat{\mathbf{u}}$ has no physiological meanings. From Table $1 \& 2$, we found that accuracy of stimuli location has some correlation with the physical locations of stimuli in the myocardium: anterior, inferior and apical stimuli can be localized with the highest accuracy because of the coverage of recording leads on the front and back of the body surface, followed by stimuli in lateral and septal region. Compared to LV stimuli, localization of RV stimuli is more difficult with an average error of $2.44 \pm 4.34 \mathrm{~mm}$, most likely because they are more hidden from body-surface recordings. Similar reasons apply to the accurate localization of epicardial stimuli, followed in accuracy by intramural and endocardial stimuli. 
Table 2. Accuracy of Single Stimulus Localization at 3 Transmural Layers

\begin{tabular}{|c|c|c|c|}
\hline Segment & Epicardial $(\mathrm{n}=55)$ & Intramural $(\mathrm{n}=59)$ & Endocardial $(\mathrm{n}=61)$ \\
\hline Error $(\mathrm{mm})$ & $0.18 \pm 1.12$ & $0.88 \pm 2.39$ & $1.12 \pm 2.51$ \\
\hline Missed $\%$ & $0 \%$ & $0 \%$ & $3.57 \%$ \\
\hline
\end{tabular}

Table 3. Accuracy of Multi-Stimuli Localization

\begin{tabular}{|c|c|c|c|c|}
\hline Types & $\begin{array}{c}\text { Two close } \\
(\mathrm{n}=10)\end{array}$ & $\begin{array}{c}\text { Two remotely } \\
(\mathrm{n}=10)\end{array}$ & $\begin{array}{c}\text { Three close } \\
(\mathrm{n}=10)\end{array}$ & $\begin{array}{c}\text { Three remotely } \\
(\mathrm{n}=10)\end{array}$ \\
\hline Average Distance $(\mathrm{mm})$ & $4.2 \pm 0.82$ & $68.82 \pm 12.56$ & $5.45 \pm 1.89$ & $50.67 \pm 10.34$ \\
\hline Error $(\mathrm{mm})$ & $1.09 \pm 1.82$ & $2.79 \pm 3.26$ & $4.02 \pm 3.87$ & $3.35 \pm 3.08$ \\
\hline Iteration number & 6.4 & 7.6 & 11.6 & 9.2 \\
\hline
\end{tabular}

B. Randomly Selected Multiple Stimuli: We further test the feasibility of our algorithm in localizing and differentiating multiple stimuli by setting 4 types of stimuli configurations within both the LV and RV (Table 3): 1) two close distributed with each other; 2) two remotely; 3) three close; and 4) three remotely. In total, we perform 40 experiments and parameter $\mathrm{N}$ is set to be $5 \mathrm{~mm}$. Table 3 summarizes the results that show high localization accuracy of our method at the presence of multiple excitation stimuli, and not seem to be influenced by the distance between the stimuli. As an example, Fig.2 (c) shows the true and reconstructed locations of 3 stimuli distributed at septal-endocardial and lateral-intramural layers, with an averaged localization errors $=3.58 \mathrm{~mm}$.

\subsection{Real Data}

Real data experiments are performed on two porcine datasets. Case 1 is a health porcine heart, and case 2 is a porcine heart with chronic infarction. For each porcine heart, a comprehensive dataset of in vivo CARTO mapping (Biosense Webster, Inc., Diamond Bar, CA) and ex vivo DW-MR (1.5T GE Signa-Excite scanner) are provided. Here, we use the first two time frames of the epicardial CARTO unipolar electrogram and the image-derived ventricular model as input data to localize the excitation stimuli. Because the exact locations of excitation stimuli are not directly available, validations are performed by simulating transmural EP dynamics with the Aliev-Panfilov model [10] using the reconstructed stimuli locations, and comparing the resulting activation isochrone with the CARTO mapping. Fig 3 (a) and Fig 4 (a) show the epicardial isochrone maps of the healthy and infarcted heart, respectively, measured by CARTO and projected to the MR-derived ventricular model.

For the healthy heart, parameter $\mathrm{N}$ is set to be $5 \mathrm{~mm}$ and iteration times is 7 . Two stimuli are identified, one localized in anterior-basal RV and the other in apical LV. Fig. 3 (c) shows the activation isochrone simulated with sinus-rhythm excitation at normal Purkinje end-terminals for human hearts experimentally determined in [11], which almost shows an opposite conduction pattern compared 

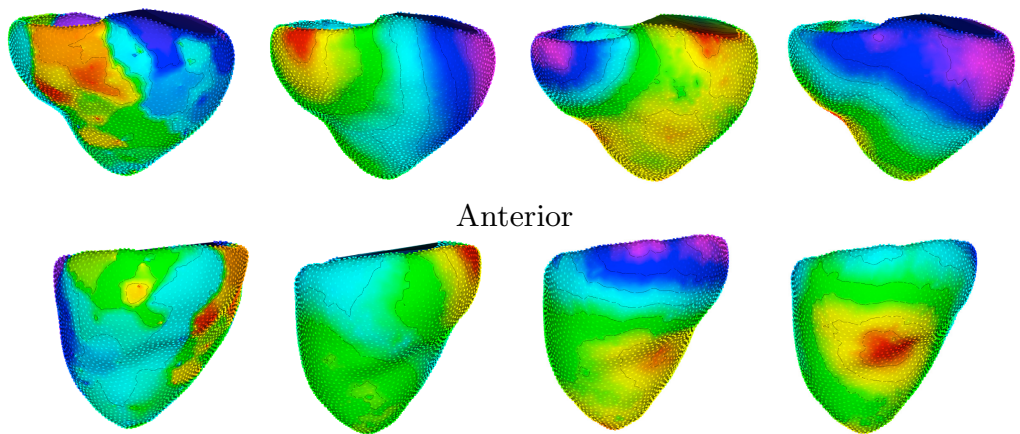

Anterior
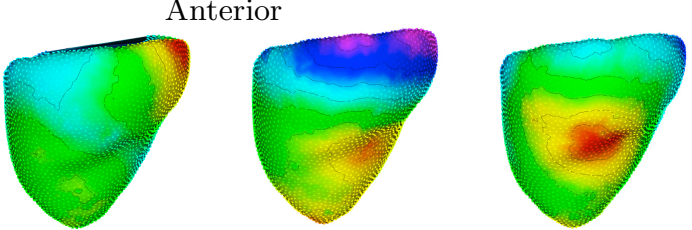

(a)

(b)

RV-Lateral

(b) (c)

(d)

Fig. 3. Epicardial isochrone maps in healthy porcine heart. (a) CARTO-mapping, (b) Simulation with reconstructed stimuli, (c) Simulation with sinus-rhythm excitation from common Purkinje end-terminals, (d) Simulation with expert suggested stimuli.

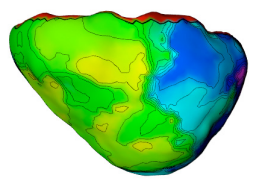

(a)

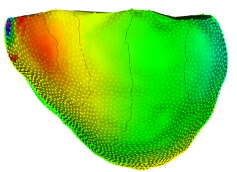

(b)

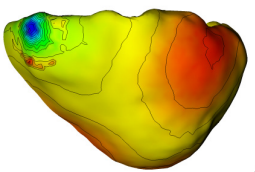

(c)

Fig. 4. Epicardial isochrone maps in the infarct porcine heart, (a) CARTO-mapping, (b) Simulation with reconstructed stimuli, (c) Simulation with sinus-rhythm excitation from common Purkinje end-terminals

to the CARTO maps (a). This may be because the porcine Purkinje structure is known to be different from human. Fig 3 (d) shows the isochrone simulated with an expert-suggested stimuli configuration based on examining CARTO maps (one stimuli at LV apex and the other at RV apex). While it captures the RV-toLV activation pattern, it fails to reproduce the local activation features especially that around RV. The averaged absolute error in activation time compared to CARTO data is $4.92 \pm 5.12 \mathrm{~ms}$. Fig. 3 (b) shows the isochrone simulated with our result, which closely captures the early activation at anterior RV as well as the RV-to-LV activation with an absolute error $3.63 \pm 2.47 \mathrm{~ms}$.

For the infarcted heart, parameter $\mathrm{N}$ is set as $5 \mathrm{~mm}$ and iteration time is 10 . The DW-MR enhanced scar is centered at middle-anterolateral LV and is incorporated into the simulation. Fig. 4 compares the epicardial CARTO isochrone map (a) with that simulated with stimuli configured at common Purkinje endterminals (error $=11.52 \pm 8.76 \mathrm{~ms})(\mathrm{c})$ and that simulated with the reconstructed 
stimuli $(7.89 \pm 5.34 m s)(b)$. As shown in the CARTO map, there is also a RV-to$\mathrm{LV}$ activation in this heart that can not be reproduced with the assumption of common excitation at Purkinje terminals, but is captured by the reconstructed stimuli at RV. As demonstrated, our method can substantially improve current practice in setting stimuli configuration by either common sinus-rhythm excitation or by experts examining CARTO maps.

\section{Discussion and Conclusion}

We presented a two-step algorithm to localize transmural excitation stimuli from surface data based on the spatial sparsity of stimuli. Phantom and real data experiments demonstrate the accuracy as well as the usefulness of this method in relevant research in subject-specific EP modeling. In the current study, stimuli localization considers only input data from one or two time instants. Future investigation will focus on the possibility to combine temporal information to the proposed sparse source reconstruction. In future studies, we will also investigate the impact of neighborhood size $N$ on the final solutions.

\section{References}

1. Zipes, D.P., Camm, A.J., Borggrefe, M., et al.: ACC/AHA/ESC 2006 guidelines for management of patients with ventricular arrhythmias and the prevention of sudden cardiac death: A report of the american college of cardiology/american heart association task force and the european society of cardiology committee for practice guidlines. JACC 48, e247-e346 (2006)

2. Wijnmaalen, A.P., et al.: Head-to-head comparison of contrast-enhanced magnetic resonance imaging and electroanatomical voltage mapping to assess post-infarct scar characteristics in patients with ventricular tachycardias: Real-time image integration and reversed registration. European Heart Journal 32, 104-114 (2011)

3. Wang, L., et al.: Noninvasive computational imaging of cardiac electrophysiology for 3-d infarct. Trans. Biomed. Eng. 58(4), 1033-1043 (2011)

4. Camara, O., Sermasant, M., et al.: Inter-model consistency and complementarity: Learning from ex-vivo imaging and electrophysiological data towards an integrated understanding of cardiac physiology. Prog. Bio. Mol. Biol., 122-133 (2011)

5. Relan, J., Sermesant, M., et al.: Parameter estimation of a 3d cardiac electrophysiology model including the restitution curve using optical and mr data. In: WC on Medical Physics and Biomedical Engineering, pp. 1716-1719. Springer (2010)

6. Camara, O., Pashaei, et al.: Personalization of fast conduction purkinje system in eikonal-based electrophysiological models with optical mapping data. In: STACOM, pp. 281-290 (2010)

7. Plonsey, R.: Bioelectric phenonmena. McGraw Hill, New York (1969)

8. Pascual-Marqui, R., Michel, C., Lehmann, D.: Low resolution electromagnetic tomography. Int. J. Psychophysiol. 18(1), 49-65 (1994)

9. Gorodnitsky, I.F., Rao, B.D.: Sparse signal reconstruction from limited data using focuss: A re-weighted minimum norm algorithm. IEEE Trans. Signal Processing 45(3), 600-616 (1997)

10. Aliev, R.R., et al.: A simple two-variable model of cardiac excitation. Chaos, Solitions \& Fractals 7(3), 293-301 (1996) 